\title{
Dealing with predators: Vigilance and alarm calling in primates
}

\author{
Phoebe Raff
}

\begin{abstract}
Predation is considered one of the most important selective pressures on animals. Evidence of predation on primates is particularly rare; however, it is thought that it has been a major selective force for many species in the primate order. This can be seen through the occurrence and application of vigilance and alarm calling in many primate species. Studies of vigilance have shown that animals have a tendency to decrease individual vigilance as group size increases. However, it has been found that primates do not fit this model, and either maintain or increase their individual vigilance as group size increases. A likely reason for the maintenance of individual vigilance is that primates utilise this behaviour as both a form of antipredation behaviour and social monitoring; thus it largely depends on the social structure of the species and the environmental conditions of the ecosystem being occupied by a primate group. Another antipredator behaviour that occurs in primates is alarm calling, which may be to deter predators. The studies presented here show that primates have the ability to distinguish between predators, between and within predator classes and also predator hunting styles. It is believed that the development of these behaviours in the primate order shows that these animals have evolved in environments where predation is a threat.
\end{abstract}

\section{Keywords}

primates, predators, vigilance, alarm calling, social monitoring, aggregation 


\section{Introduction}

While witnessed accounts are rare, predation on primates has been observed. For example, chimpanzees (Pan troglodytes) have been known to strategically hunt and kill red colobus monkeys (Procolobus badius) (Treves 1999), and howler monkeys (Alouatta seniculus) have been documented to be eaten by jaguars (Panthera onca) (Peetz et al. 1992) and harpy eagles (Harpia harpyja) (Sherman 1991). However, as such witnessed accounts are rare, it seems that the presence of antipredator behaviours may be a better way to understand the level of threat posed to primates by predators. Given their widespread nature within the primate order, the presence of antipredator behaviours may in fact indicate that primates have evolved behavioural adaptations to deal with the selection pressure of predation threats.

Antipredator behaviours come in many forms and can be utilised in different ways within and across species. While such behaviours include concealment, active defence and flight, the two forms of antipredator behaviour that will be the focus of this research essay are vigilance and alarm calling. These two antipredator behaviours are highlighted here because they are important to predator-prey studies due to their wide use among primates to deter potential threats, and they are often used to initiate further responses within primate groups. The foundations of vigilance studies have been laid out and the benefits and costs associated with individual and group vigilance have been discussed in previous works. However, it is generally thought that primates do not fit the usual models for the function of vigilance when compared to other animals because their individual alertness does not decrease as group size increases (Treves 1999, 2000). This is contradictory to the 'many eyes' and 'dilution effect' hypotheses so common in vigilance studies that suggest that with increasing group size there should be a decrease in individual vigilance (Roberts 1996; Beauchamp 2003).

Alarm calling is also an extremely important tool used by primates to signal predator threats. This behaviour is employed differently across primate species and can be used to signal threats to the group, including predator classification and to deter predators (Seyfarth et al. 1980; Zuberbuhler et al. 1997; Ramakrishnan et al. 2005). It is thought that vigilance and alarm calling have both positive and negative effects on groups and individuals, and are utilised differently across primate species. However, they primarily serve as indicators that primates have evolved with the adaptations necessary to deal with predators. 
In this research essay, I will describe both the positive and negative impacts of vigilance and alarm calling on primate groups. With respect to vigilance studies, I will discuss how primates differ from each other and the foundational hypotheses in their employment of vigilance, and how social structure, aggregational living and environment are important contributors to primate vigilance. Alarm calling is also shown to be a technique employed by primates to deter potential predators, and, like vigilance, many species use this technique differently. It is concluded that the use of vigilance and alarm calling in primates, specifically the species discussed throughout this essay, is evidence that primates have evolved in environments with predation threats and have incorporated these responses into their group structures to deal with predators and other potential threats.

\section{Vigilance}

Vigilance is defined as the visual scanning of the surrounding environment by animals, and is often assumed to be a behaviour aimed at predators. However, its employment can also be associated with the social structure of a species and their environmental conditions (Treves 2000; Beauchamp 2003). In many animal studies, it has been exemplified that increases in group size lead to decreases in individual vigilance (Treves 2000). This is known as 'the group size effect' and, according to Roberts (1996), there are two main hypotheses and associated benefits to explain the theory.

The first hypothesis is termed the 'many eyes' hypothesis, which states that individuals in larger groups should enjoy the same rate of predator detection, despite being less vigilant, because they can rely on the vigilance of others, allowing them to reduce their own alertness in order to maximise feeding time (Roberts 1996). For this hypothesis to work, collective detection must lower the individual risk of predation because if nonvigilant animals gain nothing from associating with vigilant ones, then individual vigilance should not be reduced in larger groups (Roberts 1996; Treves 2000). This is supported by Roberts' (1996:1077) suggestion that 'if vigilance depends on predation risk and if that risk declines with increasing group size, vigilance should also decline with increasing group size'.

The second hypothesis, often referred to as the 'dilution effect', suggests that an individual's risk of predation becomes diluted by living in larger groups with more potential predation targets than just themselves. Thus, under this 
hypothesis, it is expected that with increasing group size comes a decreased individual risk of being targeted by predators (regardless of vigilance rates); thus individual vigilance should decrease (Beauchamp 2003). This theory is based upon the assumption that animals in larger groups would exert the same effort into individual vigilance as animals in smaller groups if the probability of being attacked by a predator were the same (Treves 2000). For this hypothesis to work, increasing group vigilance must improve the detection of predators; otherwise individual vigilance would not vary according to risk (Treves 2000).

While the two aforementioned hypotheses present benefits to increased group vigilance against predation with increasing group sizes, there are two other things that need to be considered-both of which stem from the aggregation required for group vigilance to work. One such cost is the increased scramble competition associated with living in large groups that results from animals having to increase foraging speed and risky behaviours to maintain food intake (Beauchamp 2003). Models of scramble competition assume that resources are limited and that foragers will jostle each other and try to increase feeding rates to gain access to a greater portion of the food supply (Lima et al. 1999). Thus, when faced with increased competition, an animal will have to forage faster and as such may make riskier decisions, such as decreasing individual vigilance or searching for food further away from the protection of the group (Beauchamp 2003). This implies that reduced foraging efficiency is a key trade-off for increased vigilance.

However, many agree that primates do not conform to the trends set out by these theories. This may be due to the fact that individual vigilance in larger groups of primates may reflect both predation pressure and the needs of their complex social systems (Treves 1999). Primates will thus often remain observant even with increasing group size to increase social monitoring, whereby primates monitor the actions of their neighbours within the group (Treves 1999; Hirsch 2002), which may be needed as intragroup competition is often associated with larger groups. This may occur irrespective of predation pressure.

The idea that primates maintain individual vigilance with increasing group size due to a combination of both social monitoring and antipredation has been supported in brown capuchin monkeys (Cebus apella), where a positive correlation between the number of individuals in a group and vigilance levels has been found (Hirsch 2002). Hirsch (2002) established that within the group he studied, social monitoring was the primary driver for vigilance as 
neighbour density and predation pressure were lower in this region. He found that the time spent vigilant correlated with time spent social monitoring, in that it was more infrequent than the time spent monitoring for predators outside the groups (Hirsch 2002). However, it was also reported that in other populations of brown capuchin monkeys, predator detection is the primary driver of vigilance as predation pressure and the time spent vigilant in these areas is higher (Hirsch 2002). Whether used for social monitoring or predator detection, brown capuchins show that a positive correlation exists between group size and vigilance. Furthermore, long-tailed macaques (Macaca fascicularis) in larger groups are better at detecting potential human predators, and as a result spend more time in the lower canopies than smaller groups where access by terrestrial predators is more prevalent (Isbell 1994). This suggests that primates in larger groups, by either maintaining or increasing their individual vigilance, are better able to detect predators.

Treves (1999) studied the effect that group size has on social monitoring in red colobus monkeys and red-tailed monkeys (Cercopithecus ascanius) in Uganda. The maintenance of vigilance levels here was due to the need to both detect predators and to monitor associates. During mating periods, he found that males glance at associates more often, while females survey associates more often at times when infants are young or out of contact (Treves 1999). His study showed no evidence for an increase of within-group surveillance associated with an increase in group size, but that these primates tended to remain vigilant. This vigilance pattern likely reflected reproductive strategies within the group associated with social monitoring, as well as serving as a predator response (Treves 1999). Vigilance here was thus associated with both antipredation and social monitoring for infant protection and mate selection.

Another study done on white-faced capuchins (Cebus capucinus) found that the time spent vigilant within the group was not expressively correlated with group size, but was actually more closely associated with the number of males in each group (Rose and Fedigan 1995). In the groups studied, alpha males were the most vigilant, and applied this vigilance when the group was accessing resources and around waterholes, in areas of overlapping range and when female protection was necessary (Rose and Fedigan 1995). Male vigilance appeared to be directed at males from outside groups, rather than towards group associates and potential predators (Rose and Fedigan 1995). This, combined with the previous studies, suggests that the application of vigilance is highly dependent on the group structuring and environment of the species. Like Treves (1999), 
Hirsch (2002) and Isbell (1994), Rose and Fedigan (1995) found that group size had no effect on the vigilance of an individual primate; a primate will remain vigilant despite the size of the group (Rose and Fedigan 1995).

However, there is a negative correlation seen between group size and individual vigilance in a study done by Ducheminsky et al. (2014), who suggested that the large group size of vervet monkeys (Chlorocebus pygerythrus) led to less predator-appropriate evasion and vigilance than expected. It is thought that the differences seen between primate species in their utilisation of vigilance occurs due to the separate social grouping needs and environmental conditions of the primates being studied. These reports also show that primates are distinct from other animals in their use of vigilance, as individual alertness does not usually decrease with increasing group size, as suggested by other animal vigilance studies (Roberts 1996; Beauchamp 2003). Importantly, these studies of vigilance in primates show that they have evolved the trait to deal with both predation and potential threats within the group. The behaviour thus is associated with both primate sociality and predator avoidance, suggesting selective pressures acting from both social and predatory forces.

\section{Alarm calling}

Alarm calling as an antipredator behaviour has evolved to alert group members to the presence of possible predators. Here, I define alarm calling as any vocalisation used to alert a group to the presence of a predator, which may then lead to other antipredator behaviours and precautions, such as concealment or flight. The use of alarm calling by individuals can have numerous positive and negative effects on both the individual and the group. Alarm calling may benefit the signaller directly by initiating a protective response within the wider group, which may include concealment, flight or defence (Zuberbuhler et al. 1999). In addition, there may be an indirect benefit to the signaller by enhancing the survival chances of close kin and by facilitating social transmission to inexperienced offspring of predator knowledge (Zuberbuhler et al. 1999). This is likely why Wheeler (2008) and Zuberbuhler et al. (1999), state that kin selection and parental care are likely causes for the evolution of alarm calling. By signalling the presence of a predator, individuals can protect potential mates and they can increase their direct fitness by protecting others, and, thus, reap the benefits of reciprocal altruism (Wheeler 2008). The caller may also benefit by causing group members to coalesce around the caller, creating a potentially dangerous mob for the predator (Wheeler 2008). Therefore, there are many potential benefits for an individual to use alarm calls. 
However, there is also one main negative consequence of alarm calling. By alerting a group to the presence of a predator, that individual may identify themselves as a potential target by making themselves known to the predator (Hollen and Radford 2009). This creates a paradox in which behaving in an altruistic way when a predator is nearby results in very costly behaviour being exhibited (Zuberbuhler et al. 1999). Due to the potentially high costs of alarm calling for the signaller, the potential benefits must have outweighed the costs in many situations, otherwise we would not expect it to have evolved as a common antipredatory behaviour. The evolution of alarm calls in primates clearly shows that they have adapted to deal with conditions in which predators are threats. Studies done on numerous species of primates, including vervet monkeys, bonnet macaques (Macaca radiata) and Diana monkeys (Cercopithecus diana diana), have shown that although they are quite adept at dealing with the threats posed before them, there are slight similarities and differences in the way primates employ alarm calls.

Vervet monkeys are amongst the most extensively studied primates with regards to their alarm calling system. Seyfarth et al. (1980) observed that adult vervet monkeys have the knowledge to not only identify predator types, but also to distinguish between predator species within classes (for example, between martial eagles (Polemaetus bellicosus) and other birds). By using specific alarm calls, vervet monkeys respond according to the threat and hunting style they believe they were faced with (Seyfarth et al. 1980). Similarly, Ramakrishnan et al. (2005) found bonnet macaques can distinguish between venomous, nonvenomous and predatory snakes. The macaques responded with caution to all snake species, but pythons (Python molurus), the only snakes known to consume bonnet macaques, were the only species that elicited alarm calls (Ramakrishnan et al. 2005). This shows that bonnet macaques have the ability to distinguish between snake species, as their main predator prompted the most active response.

Likewise, male Diana monkeys are also capable of distinguishing between predators. However, their use of alarm calls is slightly different in that males only call in response to leopards (Panthera pardus) and crowned hawk eagles (Stephanoaetus coronatus), but not in response to chimpanzees and humans who will pursue the caller in the canopy. This indicates the monkeys are not only able to differentiate the species of predator, but also their hunting behaviour (Zuberbuhler et al. 1997). Furthermore, Zuberbuhler et al. (1997) also find that once the Diana monkeys made an alarm call, the males would 
approach the predator as a group. It was concluded that their calls are used to signal to the predators that rely on stealth that they had been detected. This 'perception advertisement hypothesis' is supported by observations that the pursuit of crowned hawk eagles by male Diana monkeys results in the predator's departure, and that leopards tend to abandon their hunt once they had been detected by the group (Zuberbuhler et al. 1997).

This recognition of hunting style makes Diana monkeys different from vervet monkeys and bonnet macaques, which seem to mainly identify predator type. These experiments show that many primates are adept at using alarm calls as a form of antipredator behaviour. The presence of this behaviour in these species shows that primates have adapted uniquely to deal with the specific predators they face in their environments.

\section{Conclusion}

Predation has been an important selective force for the behaviours of primates, which has been exemplified through the evolution of vigilance and alarm calling in these animals. While vigilance does not follow the same trend in primates that it does in other foraging mammals, due to their need to monitor associates, the fact that it is also associated with predators suggests it likely evolved in the company of predators. Further, primates all employ vigilance to varying degrees but, in general, they rely on each other for the detection of predators. They also monitor their associates should threats arise within the group. Alarm calling is further evidence that primates evolved in the presence of predators, and is also employed differently from species to species. It was shown that vervet monkeys and bonnet macaques have the ability to define species within classes of predators (Seyfarth et al. 1980; Ramakrishnan et al. 2005), while Diana monkeys also respond differently based on predator hunting style (Zuberbuhler et al. 1997). This is evidence that each species has evolved specific mechanisms that are likely suited to the specific needs of their own environments. Antipredator behaviours come with ecological trade-offs. For example, does one initiate an alarm call and draw attention to themselves and save possible mates and kin; or does one remain vigilant, but lose access to resources? Whether primates are conscious of the decisions they make is up for debate; however, the presence of antipredator behaviours in primates shows that they have evolved in complex social groupings and in environments alongside predators making these ecological trade-offs necessary for their survival. 


\section{Acknowledgements}

I would like to thank Alison Behie and Rebecca Hendershott, who were my course convenor and tutor for the 'Primate Ecology and Behaviour' course. Your enthusiasm for and love of primates helped to fuel my own interest in this area and in wider topics of conservation.

\section{References}

Beauchamp G. 2003. Group-size effects of vigilance: A search for mechanisms. Behav Process. 63(3):141-145. doi.org/10.1016/S0376-6357(03)00011-1

Ducheminsky N, Henzi SP, Barrett L. 2014. Responses of vervet monkeys in large troops to terrestrial and aerial predator alarm calls. Behav Ecol. 25(6):1474-1484. doi.org/10.1093/beheco/aru151

Hirsch BT. 2002. Social monitoring and vigilance behaviour in brown capuchin monkeys (Cebus apella). Behav Ecol Sociobiol. 52(6):458-464. doi.org/10.1007/s00265-002-0536-5

Hollen LI, Radford AN. 2009. The development of alarm call behaviour in mammals and birds. Anim Behav. 78(4)791-800. doi.org/10.1016/j. anbehav.2009.07.021

Isbell LA. 1994. Predation on primates: Ecological patterns and evolutionary consequences. Evol Anthropol. 3(2):61-71. doi.org/10.1002/evan. 1360030207

Lima SL, Zollner PA, Bednekoff PA. 1999. Predation, scramble competition, and the vigilance group size effect in dark-eyed juncos (Junco hyemalis). Behav Ecol Sociobiol. 46(2):110-116. doi.org/10.1007/s002650050599

Peetz A, Norconk MA, Kinzey WG. 1992. Predation by jaguar on howler monkeys (Alouatta seniculus) in Venezuela. Am J Primatol. 28(3):223228. doi.org/10.1002/ajp.1350280307

Ramakrishnan U, Coss RG, Schank J, Dharawat A, Kim S. 2005. Snake species discrimination by wild bonnet macaques (Macaca radiata). Ethology. 111(4):337-356. doi.org/10.1111/j.1439-0310.2004.01063.x

Roberts G. 1996. Why individual vigilance declines as group size increases. Anim Behav. 51(5):1077-1086. doi.org/10.1006/anbe.1996.0109 
Rose LM, Fedigan LM. 1995. Vigilance in white-faced capuchins, Cebus capucinus, in Costa Rica. Anim Behav. 49(1):63-70. doi.org/ 10.1016/0003-3472(95)80154-5

Seyfarth RM, Cheney DL, Marler P. 1980. Monkey responses to three different alarm calls: Evidence of predator classification. Science. 210(4471):801-803. doi.org/10.1126/science.7433999

Sherman PT. 1991. Harpy eagle predation on a red howler monkey. Folia Primatol. 56(1):53-56. doi.org/10.1159/000156528

Treves A. 1999. Within-group vigilance in red colobus and redtail monkeys. Am J Primatol. 48(2):113-126. doi.org/10.1002/(SICI)1098-2345(1999) 48:2<113::AID-AJP3>3.0.CO;2-K

Treves A. 2000. Theory and method in studies of vigilance and aggregation. Anim Behav. 60(6):711-722. doi.org/10.1006/anbe.2000.1528

Wheeler BC. 2008. Selfish or altruistic? An analysis of alarm call function in wild capuchin monkeys, Cebus apella nigritus. Anim Behav. 76(5):14651475. doi.org/10.1016/j.anbehav.2008.06.023

Zuberbuhler K, Jenny D, Bshary R. 1999. The predator deterrence function of primate alarm calls. Ethology. 105(6):477-490. doi.org/10.1046/j.14390310.1999.00396.x

Zuberbuhler K, Noe R, Seyfarth RM. 1997. Diana monkey long-distance calls: Messages for conspecifics and predators. Anim Behav. 53(3):589604. doi.org/10.1006/anbe.1996.0334 
This text is taken from the The Human Voyage: Undergraduate Research in Biological Anthropology: Volume 1, 2017, edited by Alison Behie, published 2017 by ANU eView, The Australian National University, Canberra, Australia.

dx.doi.org/10.22459/HV.01.2017.05 